\title{
NSIS-based Quality of Service Management in IMS Network
}

\author{
Taam Abdelkarim \\ Laboratoire Informatique et \\ Modélisation \\ Faculté des sciences Dhar El \\ Mahraz (FSDM) \\ Fès - Maroc
}

\author{
Oumsis Mohamed \\ Laboratoire Informatique et \\ Modélisation \\ Faculté des sciences Dhar El \\ Mahraz (FSDM) \\ Fès -Maroc
}

\author{
Raouyane Brahim \\ \& Bellafkih Mostafa \\ Département de Mathématique \\ et Informatique Faculté des \\ Sciences Aïn Chock \& INPT \\ Casablanca-Rabat-Maroc
}

\begin{abstract}
The next generation network requires a suitable QoS that can increase number of users and revenues. The IMS (IP Multimedia Subsystem) import new taxation strategy flow based and QoS management policy-based. The QoS management poses a competition in terms of limitations of physical resources and the onset of congestion, which encourages more organisaton to look for management solutions other than DiffServ and IntServ. The NSIS (Next Steps in Signaling) appears as a magical solution that couples DiffServ and IntServ with other properties of control traffic to validate these properties and shows the integration with IMS we present this article. Our goal, in this article, is give an idea about a Framework that coupling NSIS and policy-based management in IMS, and experimentally verify results.
\end{abstract}

\section{Keywords}

IMS (IP Multimedia Subsystem); QoS(Quality of Service); DiffServ(Differentiated Services); InterServ(Integrated Services);NSIS(Next Steps in Signaling).

\section{INTRODUCTION}

The IMS (IP Multimedia Subsystem)[1], is a NGN main layer, liaises control allows an agnostic access with all customer equipments. IMS is an essential and complementary subsystem for network service, its coexistence with other components such as the access layer performs the features for next-generation architecture to provide multimedia services. The IMS is considered as a reference, that operators build functional elements according to its specifications; it also defines the underlying standards, including standards for security, quality of service (QoS), and the new family of Charging flow-based.

The IMS describes three main levels: Access/ Transport, Control and Service architecture inter-operates with multiple types of access networks, Mobile IP, Broadband xDSL, cable and wireless LAN IP, PSTN (Public Switched Telephone Network).

The description of the main and the protocols used on the various communication interfaces open several traditional questions of the internet such as mobility, security, and also the QoS management entities. However, the aspect of QoS management [2] is the first step to differentiate the IMS classic internet. The IMS has a control plane enables communication with the other two planes and Access Service; it allows selecting multiple services and applications. Thus, the control plane ensures QoS negotiation scenarios with dynamic signalization. These scenarios outperform existing limited and developed for the internet at the same time the mechanism models inherits the famous model of QoS management for IP network like IntServ[3] and DiffServ[4] To differentiate the models, it is necessary to present the aspect of QoS management in an IMS network. This will be the purpose of the following article will detail the aspect of QoS management with a new integration of NSIS [5] protocol and submit a Management Framework based on 3GPP standards.

\section{QOS IN IMS NETWORK:}

The Access / Transport underlying layer must be notified to perform an adequate reservation services or sessions. The IMS interacts with transport layer to transmit all requirements in terms of QoS and resources. The architecture of new generation (NGN) includes separate entities, which are responsible for QoS management and charging functionality of resources. These entities employ two modes of interaction: Pull or Push, and allows Access/Transport layer to choose a mechanism to manage QoS that can be DiffServ or InterServ or new technology NSIS.

\subsection{QOS IN IMS:}

The management is performed via service requirements translation in an understandable and applicable policy by Access Layer / Transport, and adds a new concept of taxation flow-based.

The concept of QoS management [6] is based on three main entities:

- $\quad$ Application Function (AF): integrated in P-CSCF that opens a channel of communication with the transport layer function, P-CSCF sends its needs for each session requested to a decision-making via Diameter protocol, QoS parameters are provided with SIP / SDP between the P-CSCF and the client.

- Policy Decision Function (PDF)/ Policy and Charging Rules Function (PCRF) : an autonomous decision entity that stores a set of policies to install / update / delete in network components, based on information received from $\mathrm{AF}$, the entity sends a policy decision and that means the configuration for a specific session to transport layer.

- Policy and Charging Enforcement Function (PCEF) /Policy Enforcement Point (PEP) : Depending on network size, the number varies from one or more, the agent resident in one router at generic case, this agent applies policy from decision-making and it map to a model of network management: Differentiated Services (DiffServ), IntServ (Integrated services), NSIS (Next Step In Signaling), MPLS-TE (multiprotocol label switching-Traffic Engineering) 
[7]. The PCEF communicate with PCRF via COPS or Diameter.

The Service Based Local Policy (SBLP) and Flow Based Charging (FBC) architectures each one provide a set of data flow filters and rules or associated instructions related to with the gateway (GGSN for example). The Gateway (GW) then uses filters to perform a control policy and charging functions based on flow, respectively. To optimize the management of IP packet filters in the gateway, it is possible for the PCC (Policy and Charging Control) architecture to provide a unique set of filters to the bridge would be used for political control and billing flows-based (Figure 1).

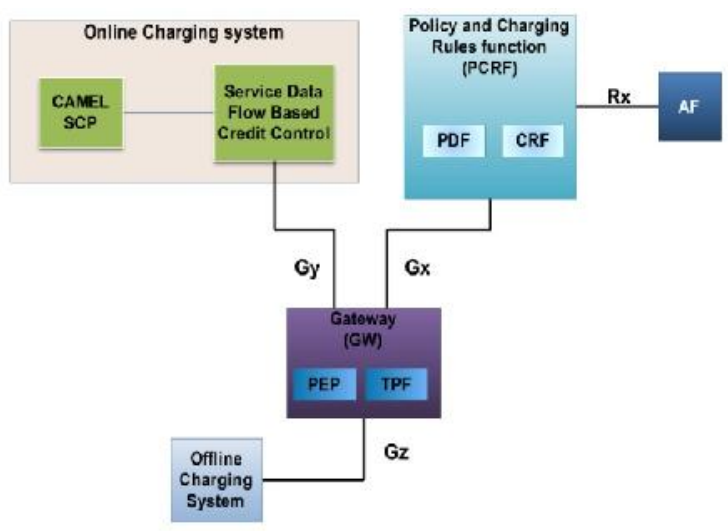

Figure 1. Reference points $R x$ and $T x$ in architecture Policy and Charging Control (PCC)

The architectures SBLP and FBC each provide an interface for application functions, so that AFs can provide information about services that are input to policy control and charging flow function.

\subsubsection{Pull mode}

The signaling network triggers a QoS authorization process following a request comes from the end user or a local event in NE(Network Element) according to preconfigured policies; indeed authorization decisions are produced at NE request.

In Pull mode, NE (Diameter client) initiates a session authorization to communicate with the licensing entity AE (Diameter Server), with two messages that are exchanged QAR (QoS-Authorization Request) and QAA (QoSAuthorization Answer) [8]. A Session, in Pull mode, begins with a request for QoS reservation or a local event received by a NE, which causes an opening of a session authorization Diameter QoS (Figure 2).

The most important information returned by the authorization entity is duration of authorization within QAA response. The life of authorization allows NE to determine time of authorization for this particular QoS reservation. To make an extension of this period, a new session with QAR/QAA messages should be launched

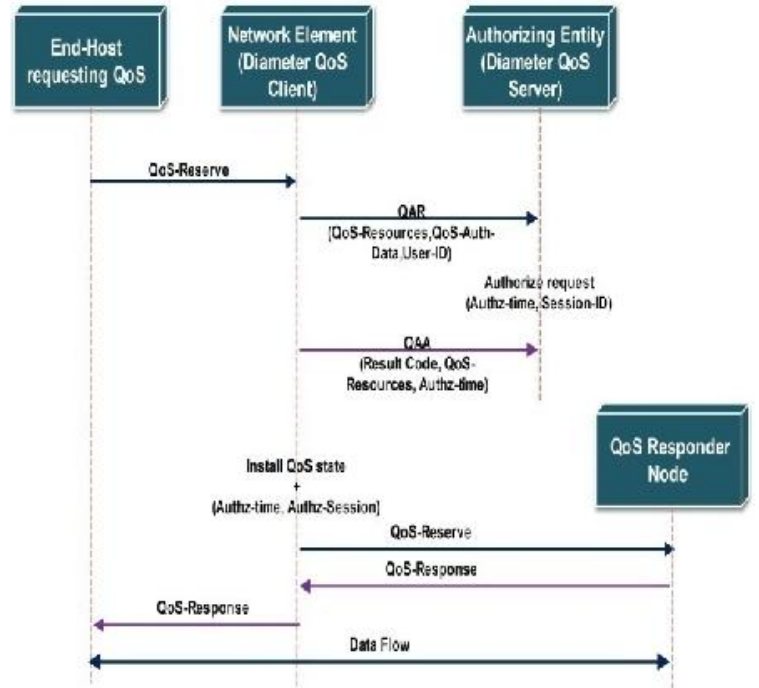

Figure 2. Pull mode and the interaction between EH, NE and $\mathbf{A E}$

A set of factors may influence the duration of authorized session, such as subscription plan of user or bills currently available to user's account and SLA (Service Level Agreement). The QoS-Reserve and QoS-Response messages are for reservation as well as RSVP protocol.

\subsubsection{Push mode}

The authorization process is triggered by QoS AppSs or local network conditions, and the authorization decisions are installed from $\mathrm{AE}$ to $\mathrm{NE}$, on its own initiative without an explicit request. To support the push mode, the AE (Server Diameter) should initiate a session authorization to communicate with the NE (Client Diameter) with no preestablished by the two main messages connection QoS-Install Request (QIR) and QoS-Install Answer (QIA) [8].

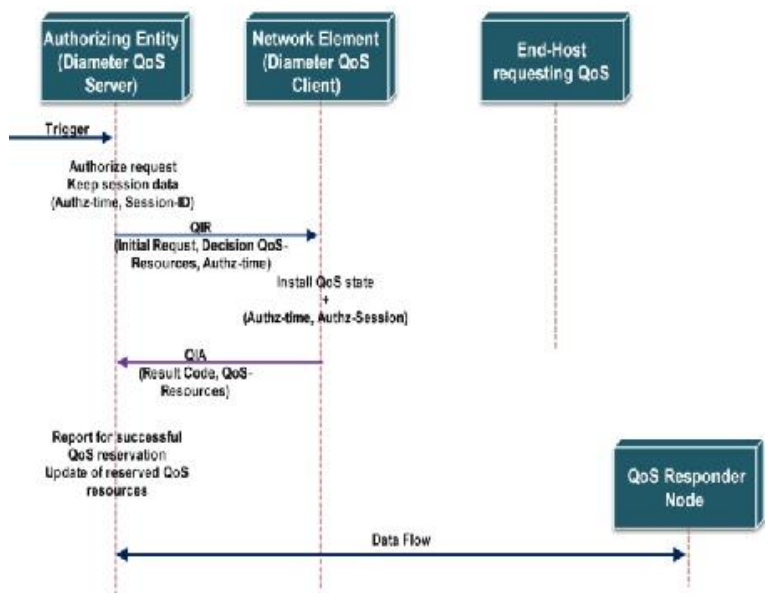

Figure 3. Push mode and interactions between AE, NE.

During a session in Push mode, the QoS-Diameter server in the $\mathrm{AE}$ initiates a session to authorize the QoS demand, signalling with the application layer or local events can trigger a QoS reservation, and generate QIR message to the client in the NE, by mapping objects required(figure 3 ).

The AE maintains session state and stores additional information for session management (eg., Signalling-SessionID, authentication data). The final result of the authorization 
decision is provided in the QoS-Resources AVP QIR message sent by the RA. The QoS information provided through QIR is installed by the traffic control function QoS (TCF) of the $\mathrm{NE}$ or can be transported to other nodes.

\subsection{Next Steps in Signalling NSIS}

Using RSVP is still limited, the IETF decided to rejuvenate and especially to overcome the inherent problems of IntServ model. The NSIS (Next Steps in Signalling) could be just RSVP v2, but the model in two layers of signalization has made it much more powerful and flexible.

GIST [9] (General Internet Signalling Transport) provides and ensures routing of signalling packets from a source until a destination via a set of routers. Unlike RSVP, all routers are not required to support protocol. As RSVP, this signalling is "On-Path" in sense that it follows the same path as data even if all routers do not participate.

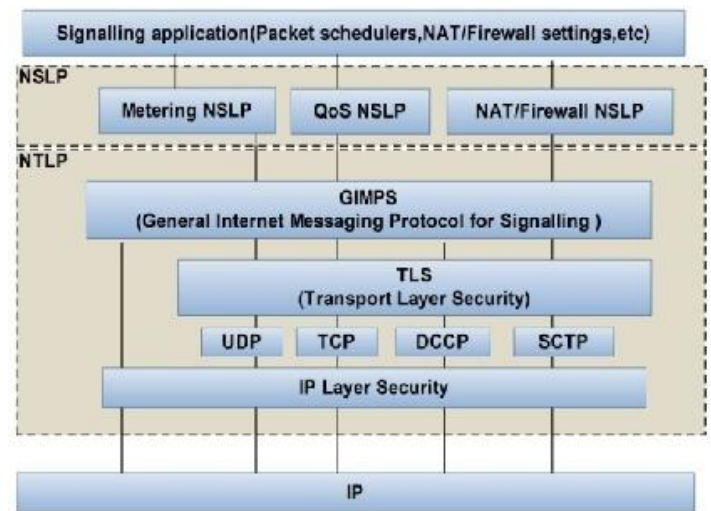

Figure 4. Protocol stack of NSIS

In protocol stack, above GIST, several so-called signalling application. NSLP (NSIS Signalling Layer Protocol) are being standardized, QoS managed and Firewall NSIS is an integral part of distributed signalization family and used in the invocation process.

In addition, the working group has defined how to use NSIS within a DiffServ domain by a control based admission and pre-emption of traffic. The latter can also be classified in the family of NSIS centralized signalling.

The NSIS protocol consists of two layers which are the NTLP and NSLP signalling layer:

- NSLP (NSIS Signalling Layer Protocol) is the top layer of protocol stack of NSIS. It is designed for a particular application signalling and has two main points of interaction. It interacts on one side with the NTLP, and another with the appropriate application signalling, which is not actually part of the NSIS protocol. In addition, NSLP can define such message formats PDU (Protocol Data Units) message sequences, etc. NSLP is application-specific signalling.

- $\quad$ NTLP (NSIS Transport Layer Protocol): is the lower layer of protocol stack NSIS. It is designed to interact with IP layer and with different NSLPs. Its role is to transport signalling messages sent by the NSLP layer between two adjacent nodes NSIS. In addition to these signalling messages, the NTLP allows the exchange of information other control such as error messages and rerouting messages.
The QoS-NSLP [10] must interact with traffic block in router and the working model of QoS-NSLP is given by figure 5 . QoS-NSLP functional design is substantially similar to the resource reservation protocol (RSVP) with better performance. The QoS-NSLP is not compatible with RSVP, but interoperability between the two protocols could be possible signalling gateways.

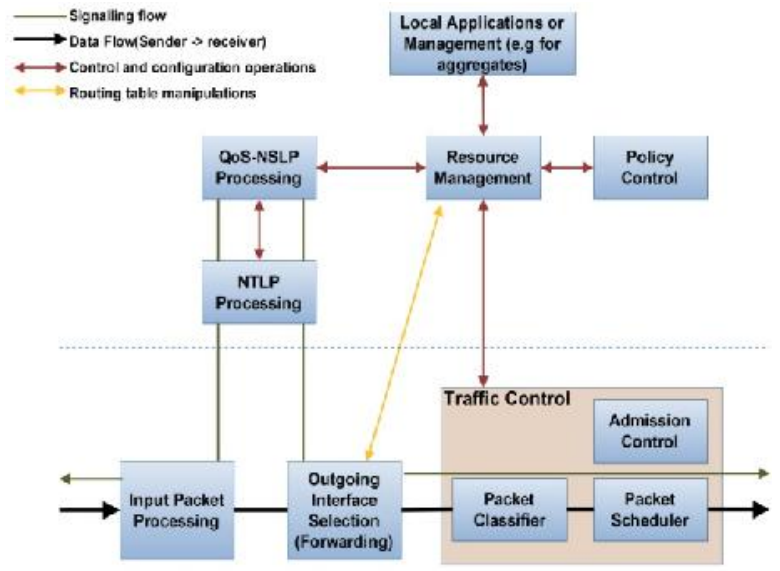

Figure 5. NSIS operating model in a router

The famous model adapted by several destinations and internet network is DiffServ, The IMS has the choice between several recent or traditional QoS management technologies, especially using the Internet model and inherited experience in QoS, but the requirements to provide multimedia services in competition to attract many customers and service providers. The appearance of NSIS can combine DiffServ and IntServ can be seen as RSVPv2 but a more interesting version of the communication model and its advantages compared to RSVP. And the question is that described model is necessary and sufficient?

\section{QOS-NSIS FRAMEWORK}

Service delivery in IMS is based on policy management with other implementation mechanisms such as DiffServ, InterServ, regarding type of infrastructure or transport layer. In addition, coupling Diameter-NSIS for QoS management provides adequate reservation depends on the logically and physically transport architecture; it can control all types of traffic and IMS and non-IMS and fast detect of flow behaviour in each node.

The architecture requires distinguishes three levels (IMS, QoS, Traffic), each plan includes a set of physical and logical entities (Figure 6).

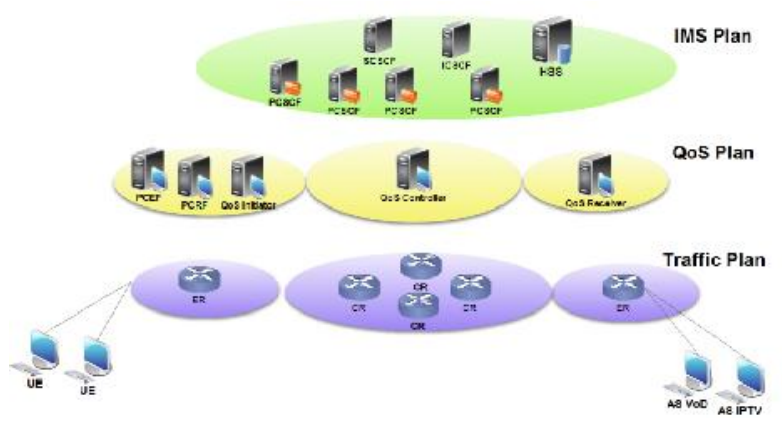

Figure 6. Communication and signalisation paln in IMS network 
The features of each plan are described as follows:

- IMS Plan: This plan to control access to IMS services, it includes usual entities in an IMS network CSCF (Proxy, Interrogating, and Server) and HSS (Home Subscriber Server). The plan may include a set of P-CSCFs to manage a large number of users and sessions. The main protocols are those signalling: SIP and Diameter. Both protocols provide internal signalling between IMS entities and external with the plan or QoS traffic. SIP communicates user needs by SDP, and Diameter executes these needs in terms of resource via policy announcing to PCRF.

- QoS Plan: is designed to provide QoS management end to end; the plan contains all NSIS entities: Initiator, Controller, Receiver, and QoS authorization entities PCRF and PCEF. The plan, to extract needs of each session or service communicates with the both plan IMS and traffic with two modes: push and pull. The QoS plan is session descriptions-based as a policy to install via PCRF and executed by PCEF. This initializes the QoS initiator with the new session parameters relative to the mechanism used (DiffServ, IntServ). The plan provides QoS with the QoS Controller agents a control policy to apply until QoS receiver.

- Traffic Plan: is a set of physical devices that convey data and signalizations. The main role of the plan is treatment of traffic with Traffic Status Block (TCB) in each router, which enables associates operations Classification, Pre-Queuing, Queuing and Scheduling, Post-Queuing for traffic.

The QoS management scenario NSIS-based should benefit from existing IMS signalizations, it uses the management policy to provide authorization to resources, in our case it is always assumed that the session was made following a pull mode (Figure 7), the mode is the most widely used to reflect reality and classical case in Internet.

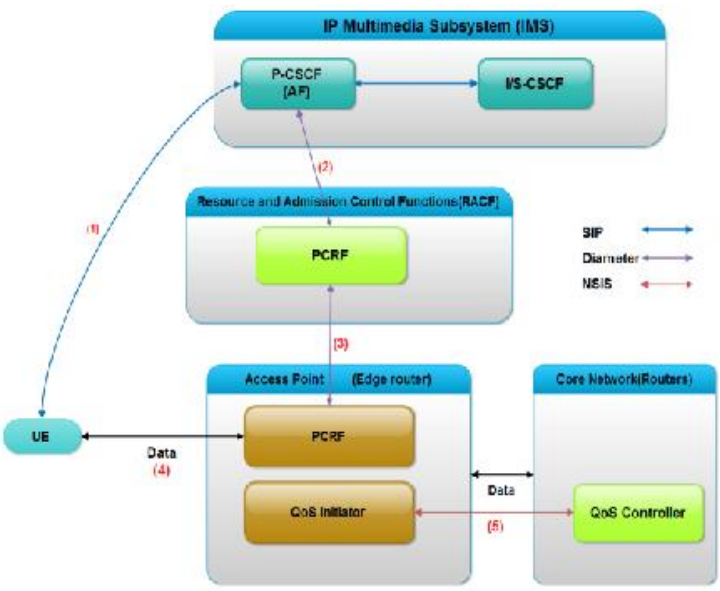

Figure 7. Communication scenarios for QoS management NSIS-based

The steps of service delivery enable interaction with tree plan: IMS, QoS, traffic in pull mode following:

(1) Initializing a session with a UE with SIP / SDP, the request contains the usual description of the session in terms type of service (codec, bandwidth and QoS), and it may include supplementary descriptions of QoS[11]. The description of the session allows to IMS network to specify session parameters of UE it configuration and related SLA (Service Level Agreement) [12] with PCRF consultation.

(2) The IMS network via P-CSCF Proxy communicates PCRF QoS-requirements, and network can interact with the UE in order to change a one or more parameters in session, the PCRF configuration is done in Pull mode with Diamater protocol, this will executed after consultation of IMS network status and installed reservation policy.

(3) The authorization is obtained from consultation PCEF; that authorization shall apply a received policy from PCRF. The PCRF/PCEF communication via Diameter protocol is to exchange information about available resources and service parameters and the translation into policy.

(4) Sending or receiving data is done after negotiation; the data must pass through a block routing and QoS processing (traffic Control).

(5) The traffic flow is controlled during the session by the NSIS nodes and it mechanisms.

\section{FRAMWORK EVALUATION}

The framework is evaluated in a test bed, which is a set of physical entities that are mentioned plans to screen (Figure 8):

- All routers (Core and Edge) that support NSIS, DiffServ and RSVP.

- Network is placed in a core router that contains IMS signalisation entities: P/I/S-CSCF and HSS in OpenIMSCore solution [13].

- PCRF/PCEF provides control policies and authorization to resources.

- Routers have all the functionality of NSIS [14]: intiator, Controller and Reciever.

- A set of IMS Client, and beside two type of video servers IPTV and VoD which are consumer of bandwidth.

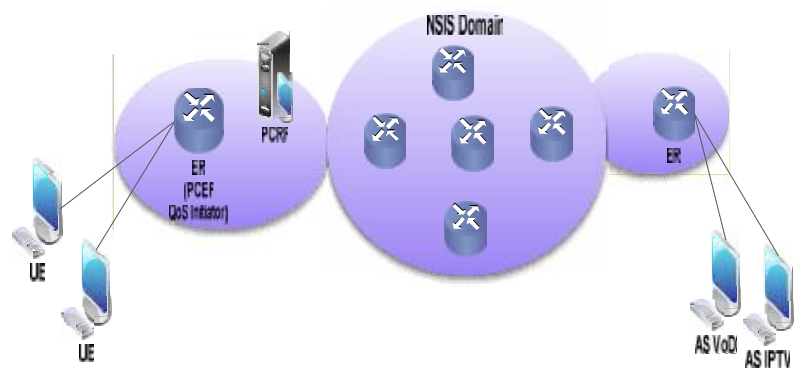

Figure 8. Test bed evaluation of QoS management in NSIS -based in IMS

The test bed is placed in a condition of overload and congestion to study the behaviour and verify some properties of NSIS. Thus, for study the QoS management coupling with policy and NSIS. This assessment also compares the results of traditional mechanisms (DiffSrv, RSVP, MPLS) vs NSIS.

\section{CONCLUSION}

An IMS network gives a standard platform for providing best multimedia internet service. The platform adds significant terms for the end customer as QoS management and flowbased billing. The QoS is a cornerstone for judging sucked 
and differentiate IMS other conventional platform. IMS uses a QoS management based on policy, and through conventional models IntServ or DiffServ policies are translated with respect to the architecture and infrastructure of transport layer. The NSIS inclusion as a model for QoS management provides several advantages and develops control QoS plan. The QoSNSLP also supports a number of mechanisms that can enrich the most important QoS management is its ability to control traffic in each node. NSIS also allows Session binding that imposes a relationship between sessions, this information can be used for local optimization in the case of bidirectional reservations or global; QoS NSLP supports facilities like aggregation. The NSIS adds an important property is detection rerouting; QoS-NSLP is able to detect route changes and automatically adapt new routes. The last property that can enrich the control plane QoS in IMS is that QoS-NSLP does not require that each node must store the NSLP reservation state QoS. It supports a "reduced state operation," as in management of resources in DiffServ (RMD), which can be used to signalization for DiffServ routers heart.

\section{REFERENCES}

[1] 3GPP TS 23.228 Technical Specification Group Services and System Aspects IP Multimedia Subsystem (IMS)

[2] RAOUYANE, B., BELLAFKIH, M., RANC, D.: QoS Management in IMS: DiffServ Model. In: 2009 Third International Conference on Next Generation Mobile Applications, Services and Technologies, Cardiff, Wales, UK. IEEE Computer Society/ACM (2009)

[3] RFC 1633 "Integrated Services Architecture", September 2003 www.ictf.org/rfc/rfc1633.txt

[4] Fine, M., McCloghrie, K., Seligson, J., Chan, K., Hahn, S., Sahita, R., Smith, A. and F. Reichmeyer,"Framework Policy Information Base", RFC 3318,;March 2003.
[5] R. Hancock, G. Karagiannis, J. Loughney, S. Van den Bosch, "Next Steps in Signaling (NSIS): Framework", RPC 4080, June 2005

[6] 3GPP TS.29.213. Policy and Charging Control signalling flows and Quality of Service (QoS) parameter mapping (Release 9)

[7] D. Awduche et al, "Requirements for Traffic Engineering over MPLS", RFC 2702, http://www.ietf.org/rfc/rfc2702.txt

[8] D. Sun et al., "Diameter Quality of Service Application," http://tools.ietf.org/id/draft-ietf-dime-diameter-qos, IETF, Oct. 2009, Internet Draft.

[9] R. Bless, "An Explicit Signaling Target Message Routing Method (EST-MRM) for the General Internet Signaling Transport (GIST) Protocol," http://tools.ietf.org/html/draft-bless-nsis-est-mrm-01, IETF, Jul. 2008, Internet-Draft.

[10] J, Manner, G. Karagiannis, A. McDonald, "NSLP for Quality-of-Service Signaling", drail-ietf-nsis-qos-nsIp12.txt, October 2006

[11] J. Polk, S. Dhesikan, G. Camarillo, 'Quality of Service (QoS) Mechanism Selection in the Session Description Protocol (SDP)'; RFC 5432,March 2009

[12] Raouyane Brahim, Bellafkih Mostafa, Errais Mohamed, Ramdani Mohamed, "IMS management and monitoring with eTOM framework and composite web service" International Journal of Multimedia Intelligence and Security 2011 - Vol. 2, No.2 pp. 172 - 185

[13] Open IMS Core, http://www.openimscore.org/

[14] Institute of Telematics, "NSIS-ka - A free C++ implementation of NSIS protocols," Feb. 2010. [Online]. Available: http://nsis-ka.org/ 